\title{
Electrocatalysts for the CO2 Electrochemical Reduction Reaction
}

\author{
Minhua Shao*, Shangqian Zhu \\ Department of Chemical and Biological Engineering, The Hong Kong University of Science and Technology \\ Clear Water Bay, Kowloon, Hong Kong, P.R. China \\ *E-mail: kemshao@ust.hk
}

The ever-rising level of atmospheric carbon dioxide and limited fossil fuel reserves have driven intensive studies on electrochemical conversion of $\mathrm{CO}_{2}$ into value-added chemicals and fuels. However, several bottlenecks have hindered the wide adoption of this technology, especially the unsatisfying performance of catalysts, which have led to the great effort on searching and developing high-performance catalytic materials. In this study, Pd-Au based nanomaterials with a unique core-shell and grain boundary-rich structure are developed. Compared with Pd nanoparticles, these materials have a significantly improved CO selectivity. A maximum CO faradaic efficiency of $94.3 \%$ (at $-0.6 \mathrm{~V}$ ), and an extremely low overpotential of $90 \mathrm{mV}$ for $\mathrm{CO}$ formation with a faradaic efficiency of $8.5 \%$ can be achieved. Combined in situ infrared spectroscopic studies and density function theory calculations reveal that surface $\mathrm{CO}$ could be more facilely generated at much lower overpotentials. 